Copyright (C) 2014 IEEE. Personal use of this material is permitted. Permission from IEEE must be obtained for all other uses, in any current or future media, including reprinting/republishing this material for advertising or promotional purposes, creating new collective works, for resale or redistribution to servers or lists, or reuse of any copyrighted component of this work in other works. 


\title{
A PACS alternative for transmitting DICOM Images in a High Latency Environment
}

\author{
Shanmugam Veeramani ${ }^{1}$, Muhammad Nasir Masood ${ }^{1}$, Amandeep S. Sidhu ${ }^{1,2}$,SMIEEE \\ ${ }^{1}$ School of Engineering \& Science, Curtin University, Sarawak, Malaysia \\ ${ }^{2}$ Faculty of Health Sciences, Curtin University, Perth, Australia \\ asidhu@ieee.org
}

\begin{abstract}
Picture Archiving and Communication System (PACS) is responsible for storing Digital Imaging and Communication in Medicine (DICOM) images from radiology modalities into its database, images takes a lot of time to transfer to remote location through WAN due to large file size and slow transfer protocol. A PACS alternative system has been developed which performs basic functions of a generic PACS. Images directly from modalities are large in size by default transfer syntax of these images is Endian Explicit syntax. Changing this transfer syntax to lossless JPEG 2000 decreases the file size and because of lossless compression quality of image is still same as original image. These compressed images are then copied into Network Attached Storage working as PACS alternative. A series of test conducted in lab with multiple transfer protocol on Network Attached Storage (NAS) to find out which transfer protocol is faster under moderate speed and high latency network.
\end{abstract}

Key words: PACS, DICOM, FreeNAS, JPEG2k, WAN.

\section{Background}

In hospitals all diagnostic procedures were film based before but as technology changed images from radiology modalities are filmless now and stored in central database digitally. DICOM is a standard introduced by American College of Radiology (ACR) and National Electrical Manufactures Association (NEMA) in 1985, which is responsible for handling, storing, printing and transmitting information in medical imaging [1]. It includes a file format definition including attributes such as patient (name, sex, date of birth), physician (name, reference number), equipment (name, model, setting) and high resolution images which make these DICOM images large in size [2].

PACS is responsible for storing these DICOM from modalities to local database and transferring those images with in LAN and over WAN by DICOM Communication Protocol [3]. Those images send to other hospitals and medical institute for study and diagnostic purposes. Images send to remote locations/hospitals through WAN, because of moderate speed and high latency, these high resolution images take time to download on destination and more time uploading imaged back to server as file size might be increased because of notes added by radiologist or consultant physician with original image having diagnostic results and treatment suggestion. [4]

Hospitals usually connected to each other with moderate speed WAN connection for transferring data between server and client. In theory Transfer time $=$ File size $/$ Network bandwidth so if the file size is big it will take more time to transfer it on other side [5]. Increasing network bandwidth could reduce file transfer time but on enterprise level leased lined cost a lot of money. So to transfer DICOM images to destination faster images are compressed to reduce file size. A NAS is designed as PACS alternative to store DICOM images and then images are compressed to reduce size so it will take less time to reach on destination and NAS server able to store more compressed mages.

\section{Methodology}

DICOM images developed from modalities are large in size, by default their transfer syntax is Endian Explicit. A CT scan of brain contain 275 sliced images is approx. $67 \mathrm{MB}$ in size. Three dimensional radiology images are approx. $1 \mathrm{~GB}$ in size or may be more [6]. Multiple DICOM images belong to different patients required more space to store on server. It's not that one file contains one attributes section and pixel data, For example 275 sliced images are extracted into a folder then every slice has same attributes information and different pixel data [9]. Therefore in some scenarios only one specific sliced DICOM image sent to some physician for study and analysis, then there is no need to send complete DICOM object. Every 
DICOM image itself can tell its purpose and history. As time passes and in long term planning more and more storage will be added to store more images which require more money. And these images takes more time to send from one hospital to another if they located in different cities or in different states.

To reduce file size, these images are compressed by lossless JPEG2k compression, transfer syntax of these images changed from Endian explicit to JPEG2k [7]. Since its lossless compression quality of images didn't change either the file format $(. \mathrm{dcm})$. Images can be easily viewed in default high resolution monitor having JPEG2K compatible viewer [8].

Brain CT scans DICOM image having size of 67 $\mathrm{MB}$ reduced to just 13.2 $\mathrm{MB}$ by lossless compression without changing image quality which means $80 \%$ size is reduced. Which means PACS alternative server now can store $80 \%$ more compressed images in its default storage.

Second technique was used by converting image into Extensible Markup Language (XML) a markup language which is both machine and human readable developed by World Wide Web Consortium. The first reason to convert image into $\mathrm{XML}$ is that XML is all about tags, and complex attributes of DICOM images easily sorted in tags of XML since it's is very structured language [10]. And second reason is, since XML is human readable language, for developing purpose DICOM images are converted in XML to make it easier to understand and develop it. XML file contains all the attributes of DICOM and binary data of Other Byte (OB) and Other Word (OW) element which has to add in output file [11]. And second file called 7FE00010 which is nothing but all the pixel data of DICOM. In the output XML file, this pixel data is linked in it. Size of XML file is about $3.5 \mathrm{MB}$ since it contains a lot of binary information of OW and OB. And size of pixel data named 7FE00010 is $63.5 \mathrm{MB}$, if both file size added up; it's the exact same size of original image. Although, the second technique using XML didn't reduce the file size of these images.
PC based cost effective model has been used in this research by replacing PACS with FreeNAS, as clear from its name FreeNAS is a free Unix based Network Attached Storage. Even requirements are not much and costly to implement. FreeNAS is much easy to install and troubleshoot; even a lot of online support is there. FreeNAS supports all modern day file sharing protocol, RAID and even ZFS (The Z File System), designed by Sun Microsystems is file sharing and logical volume manager system.

To replace DICOM communication protocol, $\mathrm{TCP} / \mathrm{IP}$ is used in lab testbed. Some common protocol are Hyper Test Transfer Protocol (HTTP), File Transfer Protocol (FTP), CIFS (Common Internet File System) and Network file system (NFS). These protocols are configured and tested on computer running FreeNAS to observe which protocol perform faster in high latency network.

In lab testbed, PACS alternative NAS is designed to test how DICOM images can be sent to destination even there is latency in the network. Latency is a time of a packet from sender to receiver and then back to sender, it is also called round trip time (RTT) [12]. Latency is directly proportional to distance even at speed of light. The longer the distance the higher latency will be. Higher bandwidth will not always solve problem, even the TCP limits the number and size of packets to send over destination, if destination is itself far away more bandwidth is not going to help here. Since in this project, images will be transferred through WAN, so dealing and overcome high latency in one of the biggest factor here. 


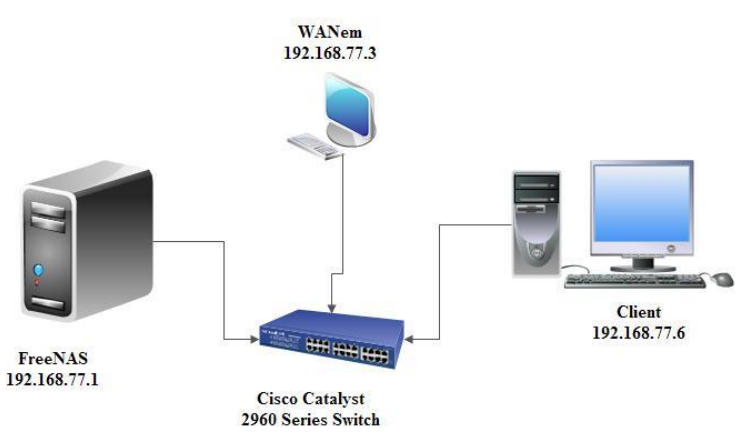

Figure 1: Lab Testbed Topology

Figure1 shows the topology designed in lab to perform various tasks. FreeNAS is installed on a Dell Workstation having Intel core 2 Duo, 500 GB 7200rpm HDD, 8 GB of RAM and accessible by IP address 192.168.77.1. NFS, CIFS, FTP and HTTP then configured on server for file sharing. Client PC has same spec as server but running Microsoft Windows XP as operating system having IP address 192.168.77.6. WAN emulator is used to create WAN like environment between server and client. WANem is accessible by 192.168.77.6 through client PC as its traffic from server routed through WANem. Cisco Catalyst switch is used to connect all three PCs.

Basic function of PACS and Network Attached Storage is same, to store data in local database and able to transfer over LAN and WAN upon request from client. FreeNAS was used in this lab testbed as it's free and easy to install and troubleshoot. All common file transfer protocols can be easily configured on it. FreeNAS also provide data security on operating system and hardware level.

\section{Results and Discussion}

Compressed and uncompressed images copied to FreeNAS server and then sent to client PC from different file protocols like NFS, CIFS, FTP and HTTP at different WAN like scenarios. On WANem latency set from $1 \mathrm{~ms}$ which is LAN latency and $800 \mathrm{~ms}$ which satellite connection latency.

Graph 1.1 shows the compressed image of file size 13.2 MB is transferred from source to destination by all file transfer protocols at different latency rate. Experiments show excellent results, NFS was the first to leave the race and on second number is CIFS. HTTP and FTP show great competition in test. Till latency $200 \mathrm{~ms}$, HTTP was winner and there is big time difference between FTP and HTTP shows in Graph 1.2. But after 200ms, ftp file transfer completed earlier. By overall performance FTP is winner here, since it's fast, easy to install and configure on FreeNAS.

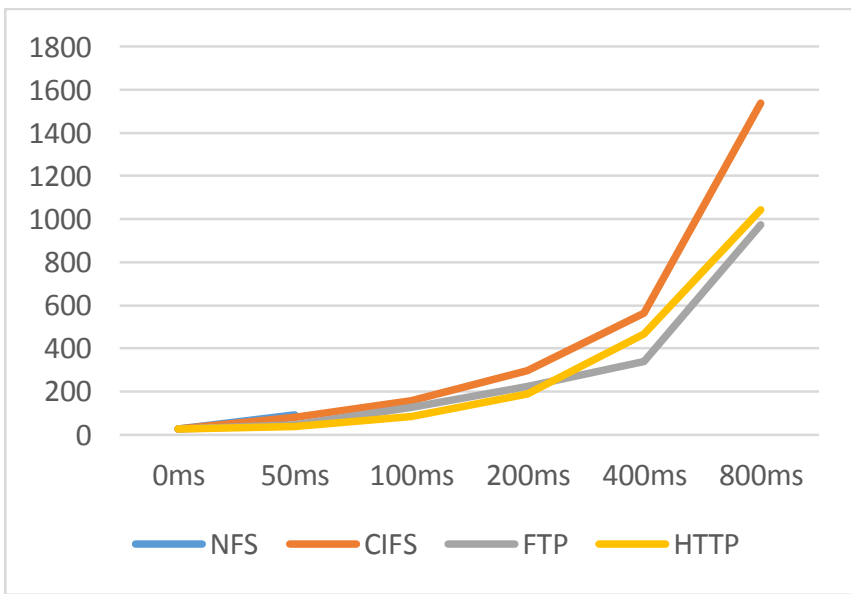

Graph 1.1: Compressed image (13.2 MB) transfer time

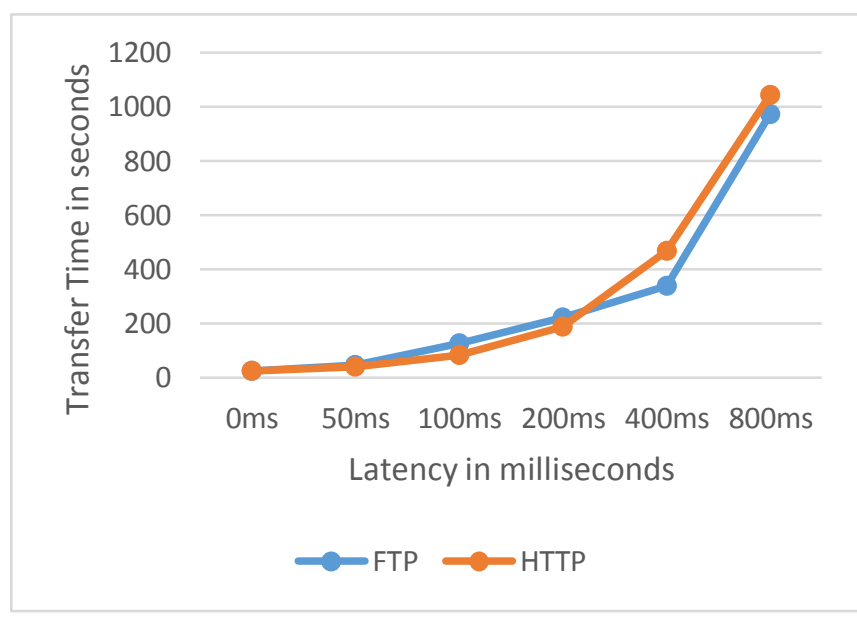

Graph 1.2: FTP vs HTTP

In Graph 1.3 below, is comparison of original uncompressed and compressed images with each other by FTP. 


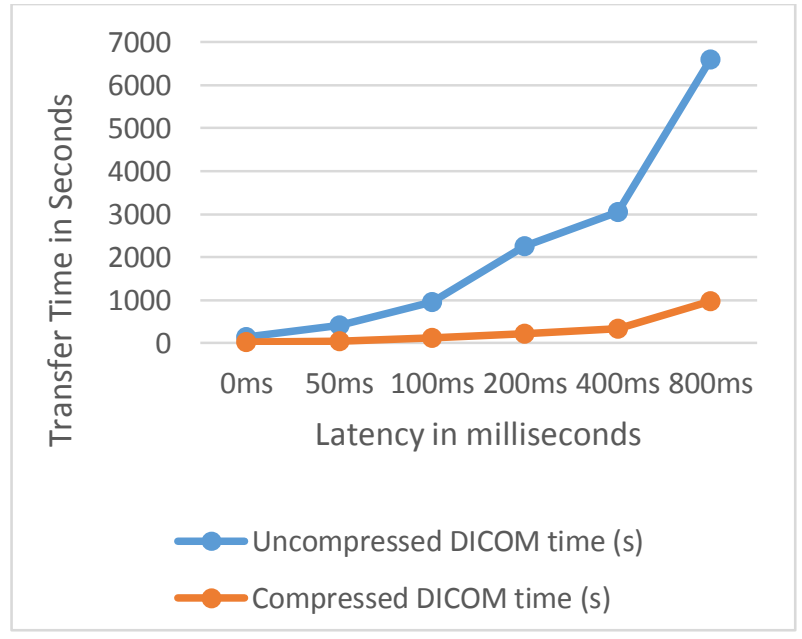

Graph 1.3: Transfer time of Uncompressed DICOM and Compressed DICOM images by FTP

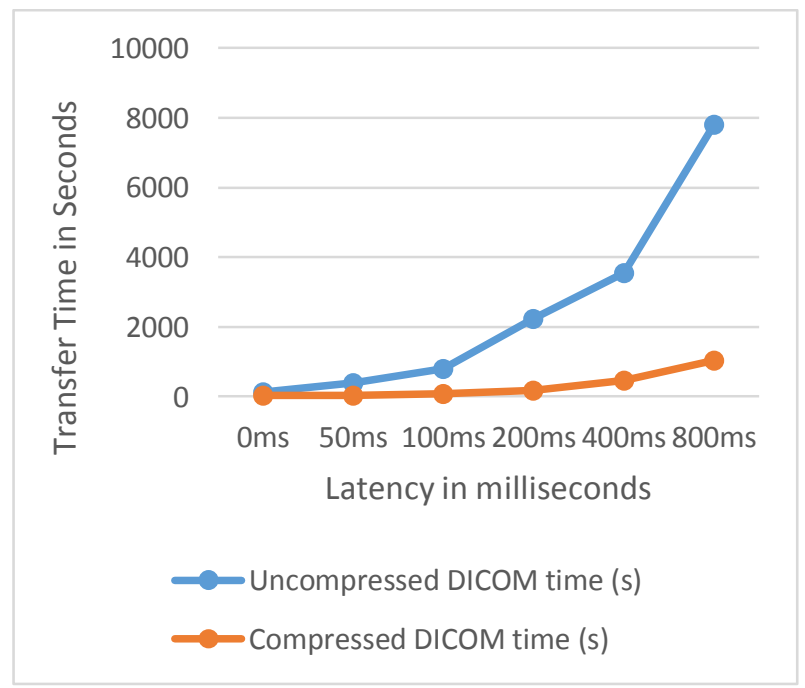

Graph 1.4: Transfer time of Uncompressed DICOM and Compressed DICOM images by HTTP

Graph 1.4: above shows transfer time of original DICOM image and compressed DICOM through HTTP. First is the huge reduction in file size. On server side, big and powerful processors are there to archive and storage, these processor can do some extra work and while storing images in archive processor compress these images so that more and more storage will available for more images. Since the file size is really small now, these images can send anytime of WAN. It is even more convenient on client end, since there hard disk is limited to some GBs, so compressed images consume less space in their PC. One the other uncompressed images definitely consumed more storage than lossy jpeg $2 \mathrm{k}$ compressed images and takes much more time to transfer over WAN. Only FTP and HTTP results comparison is demonstrated here, since both are fastest among all.

By comparing Graph 1.3 \& Graph 1.4, it is cleared the compressed images transferred in very less time as original images.

These tests are performed as clients were using a 4 Mbps broadband connection to download data. In a scenario where client are facing slow internet connection, test results will be different. Like some consultant physicians, when they are away from their offices they need to access archive database for study or diagnostic purposes so they can $3 \mathrm{G}$ connection on their tablet computers.

Like in lab test environment, client was using 4 Mbps connection, in this connection theoretically $\max$ download speed is $525 \mathrm{~KB} / \mathrm{s}$. Yes in high latency these numbers won't make much difference but in a situation where latency is more than $400 \mathrm{~ms}$ and using FTP file average downloading speed is $180 \mathrm{~KB} / \mathrm{s}$, now if using a 1 Mbps connection (broadband or $3 \mathrm{G}$ ) where max download speed is $125 \mathrm{~KB} / \mathrm{s}$, definitely downloading slows down. If will take $4.7 \mathrm{~min}$ to download a $50 \mathrm{MB}$ file on 4 Mbps connection with $400 \mathrm{~ms}$ delay but it will take $6.8 \mathrm{~min}$ to complete same file on $1 \mathrm{Mbps}$ connection.

Even a password protected zip file containing JPEG2000 compressed image is used to transfer between client and server. The reason for using password protected zip file is that usually hospitals use private VPN between their server and clients to transfer data. In high latency, VPNs slows down the network speed. Instead of using private VPN, password protected zip file containing patient data transferred to clients and an email contain password to open it. By this way VPN will no more reduce performance.

\section{Conclusion}

A PACS alternative Network Attached Storage is designed to store DICOM images and to transmit over high latency WAN. FreeNAS was used and it worked well throughout the experiments. Then 
image was compressed by JPEG 2000 to reduce size and as an advantage, image quality was same as original. DICOM protocol was replaced by TCP/IP to gain efficiency and fast transmission through Internet even if too much delay is there.

So in the lab testbed, after creating WAN environment by using WANem, some common file sharing protocol were used and tested to download large data on client computer. NFS share stop responding when delay was over $100 \mathrm{~ms}$ between server and client but CIFS worked well till last but not as fast as FTP and HTTP. Graph 6.1 clearly shows that both protocol were close to each other till $200 \mathrm{~ms}$ delay, after $200 \mathrm{~ms}$ FTP was faster than HTTP. And Graph 6.2 \& 6.3 shows that when file size increases with delay, FTP finished download earlier with good average downloading speed.

PACS is commercial licensed product, designed by specific vendors according to the demand of their specific clients which makes it much cost to install and trouble shoot. As its network attached storage alternative, system cost also reduced as FreeNAS is free and easy to use. Any moderate system administrator can able to troubleshoot it's easily as there is a lot of online support is available.

\section{References}

[1] N. Center, A Brief History of Biomedical Informatics as a Discipline, NYU Graduate Training Program in Biomedical Informatics (BMI), 2010.

[2] K. A. T. B. S. L. A. W. W. a. R. L. A. H.K. Huang, "Design and Implementation of a Picture Archiving and Communication System: The Second Time," Journal of Digital Imaging, 1996.

[3] Y. a. G. Y. Lidong, "Design and realization of DICOM/HL7 gateway in PACS. in Electronic and Mechanical Engineering and Information Technology," 2011 International Conference EMEIT, 2011.
[4] J. e. a. Pereira, "Design and implementation of a DICOM PACS with secure access via Internet. in Engineering in Medicine and Biology Society,," Proceedings of the 23rd Annual International Conference of the IEEE, 2001.

[5] W. J. Langer SG, An evaluation of ten digital iamge review workstations, J Digit Imaging, 1997.

[6] O. Pianykh, "Digital Imaging and Communications in Medicine (DICOM)," Springer Berlin Heidelberg, 2008.

[7] R. A. L. B. C. B. P. B. S. Dolin, "The HL7 clinical document architecture," Journal of the American Medical Informatics Association, 2001.

[8] S. Y. K. D. Piyamas Suapang, "A Web-based DICOM-Format Image Archive, Medical Image Compression and DICOM Viewer System for Teleradiology Application," SICE Annual Conference, 2010.

[9] W. D. V. L. J. M. A. O. S. K. CD. Stockham, "Implementation and experiences with a minimal GUI diagnostic display station," Proceedings of SPIE, Medical Imagin, 1999.

[10] A. S. T. D. L. J. S. W. D. O. N.J. Hangiandreou, "Evaluation of DeJarnette ImageShare CR Workstation," Private Communication, 1999.

[11] A. S. o. U. B. O. W. M. S. H. M. U. H.-P. M. Uwe Engelmann, "A Three-Generation Model for Teleradiology," IEEE TRANSACTIONS ON INFORMATION TECHNOLOGY IN BIOMEDICINE, 1998.

[12] M. N. B. M. e. a. Taira RK, "Design and implementation of a picture archiving and communtion system (PACS) for pediatric radiology," AJR Am J Roentgenol, 1988. 
